\title{
LA FORMACIÓN JURÍDICA DE LOS DIRECTIVOS UNIVERSITARIOS.
}

\section{THE LEGAL FORMATION OF UNIVERSITY DIRECTORS.}

Reinier Bosmenier Cruz, MSc. Máster en Ciencias de la Educación (Cuba). Coordinador de la carrera de Derecho en la Universidad de Pinar del Río, Cuba. rbosmenier@upr.edu.cu

Duniesky Alfonso Caveda, PhD. Doctor en Ciencias Pedagógicas (Cuba).

Contralor Académico.

Docente titular de la Facultad de Derecho y Gobernabilidad en la Universidad Tecnológica ECOTEC, Ecuador. dalfonso@ecotec.edu.ec

\section{ARTÍCULO DE INVESTIGACIÓN}

Recibido: 22 de noviembre de 2016.

Aceptado: 4 de enero de 2017.

\section{RESUMEN}

La formación jurídica de los directivos universitarios constituye en la actualidad una necesidad insoslayable, si se aspira a alcanzar una formación integral de estos agentes de la Administración Pública, quienes deben incorporar el Derecho a su actuar diario en el proceso de toma de decisiones, las cuales deben sustentarse en las normas, principios y valores que informan el ordenamiento jurídico, en un marco de respeto al Derecho, evitando se produzcan flagrantes violaciones a la legalidad.

La presente investigación está dirigida a analizar las principales manifestaciones del proceso de formación jurídica de los directivos en las Instituciones de Educación Superior, a nivel internacional y nacional, hasta su expresión en el contexto de la Universidad de Pinar del Río.

Palabras clave: formación jurídica, Derecho, directivo universitario. 


\section{ABSTRACT}

Legal training of university directors is now an unavoidable necessity if the aim is to achieve comprehensive training of these Public Administration agents, who must incorporate the application of law in their daily process of decision making. This process should be based on the norms, principles and values that inform a legal system, within a framework of respect for the law and avoiding flagrant legality violations.

This research is aimed at analyzing the main manifestations of the process of legal training of managers in Higher Education Institutions, at an international and national level, as well as its manifestation in the context of the University of Pinar del Río.

Keywords: legal training, law, university directors.

\section{INTRODUCCIÓN}

La formación jurídica de los directivos universitarios constituye una necesidad y a la vez una exigencia de los tiempos actuales, en la aspiración de lograr una formación integral de estos sujetos encargados de la gestión de los procesos que se desarrollan en la institución que dirigen, quienes deben incorporar el Derecho a su actuar diario durante la toma de decisiones, las cuales deben ser guiadas por las normas, principios y valores que informan el ordenamiento jurídico, en un marco de respeto al Derecho y de estricto cumplimiento de la legalidad.

El siguiente trabajo está dirigido a analizar las principales manifestaciones del proceso de formación jurídica de los directivos en las Instituciones de Educación Superior, a nivel internacional y nacional, hasta su expresión en el contexto de la Universidad de Pinar del Río.

Para ello, se abordan diferentes concepciones, enfoques y criterios, de autores y organismos, que permiten la comprensión de este proceso, y se realiza una valoración diagnóstica en la Universidad de Pinar del Río.

\section{La formación jurídica de los directivos y su carácter de proceso: fundamentos conceptuales y epistemológicos.}

El vocablo proceso proviene del latín procesus, que significa paso, avance. Según el Gran Diccionario de la Lengua Española, en su edición digital se define, como "el conjunto de fases sucesivas de un fenómeno natural o una operación artificial", refiriéndose en todo momento a la evolución por la que el mismo transita. El Diccionario Filosófico se refiere al 
proceso, como: "los fenómenos, acontecimientos, hechos, que se suceden, pasando por diferentes estados. Se define como transformación sistemática, sujeta a la ley, de un fenómeno; como el paso del mismo a otro fenómeno (desarrollo)" (Rosental, M \& P. ludin, 1981, p.376).

Para Álvarez de Zayas (1999), un proceso es "una sucesión de estados de un objeto determinado". Otros autores lo definen, como "una transformación sistemática de los fenómenos sometidos a una serie de cambios graduales, cuyas etapas se suceden en orden ascendente; como tal, solo puede entenderse en su desarrollo dinámico, su transformación y constante movimiento"(Colectivo de Autores, 1984, p.182).

El autor de la investigación define entonces por proceso: la sucesión de etapas de desarrollo de un fenómeno, orientado hacia un fin determinado. De esta noción se desprende que todo proceso debe contar con los siguientes elementos: actor, actividad, etapas y misión o finalidad. El actor es el agente, el sujeto que interacciona con otros. La actividad es lo que el actor realiza en un espacio físico concreto y durante un tiempo determinado. Las etapas se refieren a las fases en que se desarrolla la actividad del actor y la misión es la finalidad que persigue dicha actividad.

Según el Gran Diccionario de la Lengua Española, en su edición digital, la formación es "la acción y resultado de formar o formarse". Además suele identificarse con mucha frecuencia, como sinónimo de educación e instrucción.

Álvarez de Zayas (1999) concibe la formación, como: "un proceso dirigido a preparar al hombre en todos los aspectos de su personalidad". Y agrega que, "la formación se considera, como un proceso de adquisición de conocimientos, habilidades, valores, y la experiencia de la actividad creadora de los profesionales que habilitan al sujeto, para el desempeño de una determinada actividad" (Álvarez, 1999, p.11).

En la Educación Superior cubana, el término se ha empleado para caracterizar uno de los procesos sustantivos que se desarrolla en las universidades, con el objetivo de preparar integralmente al estudiante en una determinada carrera o profesión y abarca, tanto los estudios de pregrado como los de postgrado (Horruitinier, 2007).

La formación se desarrolla como un proceso complejo y multidireccional, mediante el cual se transmiten y reciben conocimientos, se adquieren habilidades, se forman valores y actitudes, y desarrollan formas de actuar. Desde esta perspectiva de análisis se puede concluir, que se trata de un proceso orientado a preparar integralmente al ser humano, para la vida en sociedad. 
La formación jurídica, por su parte, constituye un proceso mediante el cual se transmiten y reciben conocimientos, se adquieren habilidades, se forman valores y actitudes y desarrollan formas de actuar con apoyo en la Ciencia Jurídica. Se trata de un proceso, como cualquier otro proceso formativo, orientado hacia la consecución de determinado fin: la preparación integral del ser humano, para la vida en sociedad. Sin embargo, su desarrollo adquiere particularidades, no solo a partir de los sujetos que intervienen como aprendices; sino también, por su contenido, dos elementos que deben ser atendidos y adaptados a la realidad circunstancial.

No se requiere el mismo tratamiento y enfoque, cuando se trata de formar jurídicamente a individuos que van a ejercer la profesión de jurista, que cuando se forman directivos de uno o varios sectores de la sociedad, para garantizar entre otros aspectos su desempeño eficaz y eficiente en la dirección de la sociedad, o cuando se ofrece una educación jurídica a toda la sociedad, entendida esta como un saber cultural.

De lo antes expresado, se coligen tres formas fundamentales de expresión o materialización de la formación jurídica.

En primer lugar, la que se desarrolla a través de la formación de profesionales que van a ejercer una actividad laboral como juristas, desde una concepción curricular, plasmada en un plan de estudios, la cual se inicia con el ingreso a la universidad, con una edad mínima de 18 años y que tendrá una continuidad con la formación postgraduada.

En segundo lugar, la formación jurídica que se dirige a los directivos para la gestión de los procesos de su organización, la cual requiere de una formación básica primero y de una formación especializada después para el desempeño de la función directiva según el perfil del cargo.

Y en tercer lugar, desde una visión más amplia, en cuanto al número de sujetos que puede abarcar, incluyendo no solo a los futuros juristas y directivos sino a toda la sociedad a través de la Educación Jurídica, la cual puede ser desarrollada por diferentes agencias socializadoras, entre ellas, la escuela, la familia, la comunidad, entre otras.

Es de sumo interés, para esta investigación, abordar el segundo enfoque antes mencionado, referido a la formación jurídica de los directivos, teniendo en cuenta que los sujetos a los que se dirige, son directivos universitarios, y pertenecen a un sector de la sociedad, de trascendental importancia, la educación superior, los cuales tienen a su cargo la gestión de los procesos formativos (docencia, investigación y extensión) y otros no formativos como la gestión económica y de los recursos humanos en las instituciones universitarias. 
La Educación Jurídica, permite al ciudadano la obtención de conocimientos, el desarrollo de habilidades y la formación de determinados valores, lo cual le posibilita la inserción en su entorno social como ciudadano, conocedor de sus deberes y derechos, apto para actuar y transformar su realidad individual y social.

Este proceso tributa, sin lugar a dudas, a la formación jurídica de los futuros directivos, los cuales accederán a la función directiva con una formación de base, que será luego complementada con una preparación general, acorde con la actividad de dirección y posteriormente con una especializada según el perfil del cargo.

Sierra (2003) definió la Educación Jurídica en Cuba como:

un proceso consciente de enseñanza y aprendizaje, de acercamiento continuo al derecho para apropiarse de su contenido político, social, ideológico, deontológico, axiológico, normativo y conductual, en función de la defensa consciente del proyecto socialista cubano, mediante el estudio de las normas jurídicas y encaminado a la formación ética del ciudadano, sobre la base de la dialéctica de deberes y derechos (Sierra, 2003, p.48).

Esta definición antes expuesta, contextualizada en la formación inicial y permanente de maestros primarios, aporta un elemento que debe ser tenido en cuenta en la formación jurídica de los directivos universitarios, el cual se refiere al estudio de las normas jurídicas, pero que resulta insuficiente pues en la actividad de dirección se produce también un acercamiento al derecho y a su contenido, mediante la creación de las normas jurídicas, en virtud de la potestad reglamentaria que le es legalmente conferida, dando nacimiento a reglamentos y disposiciones internas (instrucciones, órdenes y circulares) y mediante la solución de los casos que requieren atención jurídica, durante la interpretación sistémica de la norma y la determinación de la que resulta aplicable o la utilización de métodos de integración para resolver posibles lagunas en el ordenamiento jurídico.

La categoría directivo, en el caso cubano, se identifica con la de cuadro, esta última establecida legalmente en el Decreto Ley 196 de fecha 15 de octubre de 1999, y que responde en la actualidad a la clasificación: directivo superior, directivo, y ejecutivo. Dicha clasificación reviste gran importancia, pues la investigación que se realiza está dirigida en lo fundamental a los cuadros ejecutivos, al no poseer la Universidad de Pinar del Rio, ningún cuadro directivo superior, poseer solo un cuadro directivo (el Rector), y pertenecer el resto de los cuadros a la nomenclatura de cuadros ejecutivos (Vicerrectores, Decanos, 
Vicedecanos, Jefes de Departamento Docentes y no Docentes, Directores de Centros de Estudio y Secretarias Docentes).

\section{El proceso de formación jurídica de los directivos a nivel internacional. Un acercamiento a su concepción y desarrollo en las IES.}

La formación de directivos, es una actividad, que con anterioridad a la II Guerra Mundial, no se le prestaba la debida importancia. Sin embargo, desde ese momento y cada vez con mayor fuerza, las organizaciones han comprendido la necesidad de encontrar y formar a sus futuros directivos.

La Organización de Naciones Unidas (en lo adelante ONU), ha definido la formación de directivos, como la actividad encaminada hacia el futuro desarrollo de los conocimientos y talentos de la práctica gerencial y la modificación o cambio en sus conceptos, actitudes y habilidades. Dicha formación deberá garantizarse a través de un programa debidamente estructurado, que les provea la preparación necesaria para operar y mejorar las actividades de la organización que dirigen.

Según Koontz y O'Donell (1961) los programas de formación de directivos deben reunir determinadas características:

1. La formación debe provenir de la Alta Dirección, ya que se supone que quien mejor conoce la función directiva es quien la ejerce.

2. El programa debe tener un carácter formal sin descuidar que existen modos para adecuarlo atendiendo a las características individuales de los sujetos a formar.

3. Debe abrir oportunidades para tener una experiencia variada, ya que esta es el medio más efectivo, para orientar al futuro directivo, en la comprensión de la interrelación que existe entre los departamentos de una organización.

4. Provisión de principios y de práctica, ya que teoría sola o práctica sola proporcionan una formación incompleta.

5. Reserva de elementos directivos, teniendo disponibilidad de personas que puedan llegar a ser directivos. (Koontz \& O'Donell, 1961, pp. 375-379).

Un elemento esencial, a tener en cuenta, en estos programas de formación de directivos, es el contenido, el cual deberá estar en función del tipo de organización de que se trate y del estudio de las necesidades de formación que se realice previamente. En este sentido Siliceo (1981, p.47) considera, como áreas de formación de los directivos más 
importantes, la administración general, las técnicas y habilidades administrativas, el desarrollo económico, las finanzas, la legislación, los aspectos internacionales, la mercadotecnia, el personal, la organización y la producción.

De este planteamiento se colige, que cada una de las áreas antes mencionadas no tienen que ser necesariamente desarrolladas en las distintas instituciones, ello dependerá de su naturaleza pública o privada.

Sin embargo, existe un área que no puede ser descuidada en la formación del directivo, sí se aspira a lograr su formación integral, y es el área de la formación jurídica, la cual está relacionada con el área de formación, denominada legislación, en un sentido general, pero que no puede reducirse solo al conocimiento de la legislación aplicable a la institución donde labora el directivo o la relacionada con la actividad en la que se desempeña, así como su correcta aplicación en los casos que conozca por razón de su competencia, sino que debe ser vista dicha formación desde una visión más profunda e integral del Derecho, no solo desde su dimensión normativa, como norma pura que debe ser aplicada a un caso concreto, sino además desde una dimensión axiológica, identificada con los valores que la norma de un modo más expreso o presunto consagra y/o aspira a fomentar o preservar, tales como la justicia, la igualdad, la equidad, la responsabilidad, y también, desde una dimensión social, a partir del contexto social particular en el que la norma será aplicada.

\section{El proceso de formación jurídica de los directivos en las IES a nivel internacional.}

La Universidad, como institución social, tiene sus orígenes en el medioevo, a mediados del siglo XII, alcanzando su apogeo en el siglo XIII. Dicho nacimiento estuvo enmarcado, al igual que el resto de las instituciones sociales, en un contexto socioeconómico y cultural que va a imprimirle sus rasgos fundamentales, los cuales van a estar determinados por varios factores, entre ellos, su funcionamiento, y el papel que desempeña el personal directivo al frente de estas instituciones.

A lo largo de la evolución y el desarrollo posterior de las universidades, surgen varios modelos, que van a determinar en gran medida el papel que ocupan los directivos en ella, y el lugar que se le asigna a su formación y desarrollo.

Los dos primeros modelos universitarios que se conocen son el de Bolonia y el de París y aunque con sus diferencias, dieron lugar a un gobierno universitario de carácter colegiado; según el criterio de Alpízar (2003, p.17), "no posibilitaba el establecimiento de 
jerarquías con atribuciones directivas y la preparación del personal que desarrollaba funciones directivas no era considerada". Posteriormente, aparece el modelo napoleónico y el modelo alemán, permaneciendo casi inamovible el perfil de quienes dirigían las universidades, siendo esencial la formación en la profesión. No obstante, el modelo alemán tuvo el mérito de introducir la departamentalización en las universidades, trayendo consigo un incremento del número de directivos y una mayor complejidad de las funciones que debían desempeñar, lo cual demandaba una mayor preparación para su desempeño, situación que no fue atendida como lo requerían las nuevas circunstancias.

En este sentido, se comparte el criterio de Alpízar (2003, p.18), de que los propios modelos universitarios y paradigmas prevalecientes, y el entorno relativamente estable en que se desenvolvían las universidades de esos primeros siglos de existencia son factores, entre otros, que contribuyen negativamente a la consideración de la necesidad de la formación del personal que está al frente de las instituciones universitarias.

En América Latina la creación de las universidades y su funcionamiento hasta inicios del siglo XX, estuvo muy influenciada por las universidades europeas, específicamente las españolas. En ellas, los directivos seguían cumpliendo funciones representativas, la universidad estaba al margen de la realidad social, desconectada de ella, y la falta de preparación de este personal, al no ser una prioridad en aquellos momentos, trajo consigo un atraso en el desarrollo de la universidad en Latinoamérica y la imposibilidad de llevar a cabo el autogobierno.

El siglo XX va a estar marcado por diferentes procesos de reformas universitarias, uno de ellos fue la Reforma de Córdoba, en Argentina, en 1918, hecho que fue calificado por Tünnermann (1996, p.55) como "el primer cotejo importante entre una sociedad que comenzaba a experimentar cambios en su composición interna y una universidad enquistada en esquemas obsoletos."

A pesar de haber contribuido dicha reforma al alcance por la universidad latinoamericana de un perfil particular, y dotarla de cierto grado de originalidad e independencia intelectual, sus postulados no consideraron un elemento esencial en la conducción eficaz de los cambios que se aspiraban a lograr con la misma, referido al papel de los directivos y su necesaria preparación en pos de materializar dichos cambios. Esta aspiración, tan necesaria de concretar, se vio frustrada sin lugar a dudas por la influencia del modelo napoleónico que no se logra rebasar con dicha Reforma, así como el divorcio aún existente entre la universidad y la sociedad. 
En la segunda mitad del siglo XX junto a los diferentes procesos de reforma universitaria, que se desarrollan fundamentalmente en la década del 60 en América Latina, Estados Unidos y Francia, caracterizadas por una transformación del tradicional esquema napoleónico y en otras regiones del mundo, concretados en "la introducción de los Estudios Generales, como tarea propia de la Universidad, la departamentalización de la docencia, el cultivo de las disciplinas básicas y una mayor flexibilidad académica mediante el establecimiento del sistema de requisitos y créditos" (Tunnerman,1996, p.63), son trascedentes y valiosas, las consideraciones expuestas sobre la Universidad; sin embargo, según el criterio de Alpízar (2003. p19), "resultan incompletas, al no valorarse ni hacerse alusión al rol que deben jugar los directivos para enfrentar con éxito las nuevas condiciones en que comienzan a desarrollarse las IES".

Los directivos universitarios se suceden de un período eleccionario a otro y no es preocupación su preparación real para ejercer las funciones y responsabilidades, cada vez más complejas, que asume.

En 1995 el Banco Mundial y la UNESCO elaboraron sendos documentos, que hacen referencia a las políticas de la Educación Superior. Por su parte el Banco Mundial, reconoce la universidad como parte de la problemática de la sociedad contemporánea, mientras que la UNESCO la asume como vía de solución a dicha problemática.

En ninguna de las consideraciones del Banco Mundial y las de la UNESCO, se analiza el papel del personal que está al frente de las IES en sus distintos niveles, al valorarse los complejos retos de la Educación Superior y las respuestas de estas instituciones sociales. La ausencia de este análisis y su consideración inmediata constituye uno de los retos de la Educación Superior en el mundo, como respuesta que garantice plena concordancia con los tres principios rectores declarados por la UNESCO (UNESCO, 1995), Relevancia, Calidad e Internacionalización.

En 1998 se desarrolló en París la Conferencia Mundial sobre la Educación Superior en el siglo XXI. En dicha reunión se debatió sobre la formación del personal de la Educación Superior como una misión permanente. Los principales planteamientos giraron en torno a las funciones y competencias de los profesores universitarios; sin embargo, no se trató con la importancia que debió tener el tema para los directivos universitarios, tomando en cuenta el rol que deben desempeñar estos sujetos en la gestión de los procesos universitarios y responder a las demandas de la UNESCO de garantizar Relevancia, Calidad e Internacionalización en las universidades. 
A pesar de la escasa atención brindada a nivel internacional a la formación de los directivos universitarios, desde los orígenes de la institución universitaria y hasta finales del siglo $X X$, existen algunas excepciones de países y universidades, que cobraron fuerza a finales del siglo $X X$, en medio del cada vez más convulso escenario en que se desenvuelven las IES, y han continuado su materialización en lo que va del presente siglo.

Un ejemplo de ello es la creación en 1998 de la Cátedra Unesco de Dirección Universitaria. Dicha Cátedra es una unidad de la Universitat Politécnica de Catalunya (UPC), que tiene como objetivo promover y dar soporte a las iniciativas que buscan la mejora y la innovación de la Educación Superior y de las universidades que se basan en los principios y los valores de la UNESCO. Su misión es impulsar la calidad, la innovación y el compromiso social de las instituciones de Educación Superior en el ámbito de la dirección y la gestión, a través del debate, la difusión de las políticas, la formación, los estudios, el asesoramiento y la gestión del conocimiento, y favorecer de esta manera la excelencia.

Dentro de las principales áreas de trabajo se destacan, la dirección y la planificación estratégica de las universidades; la política universitaria y el financiamiento de la Educación Superior; los sistemas innovadores de organización y de gestión de las universidades; la evaluación de la calidad universitaria; la dirección y gestión de la docencia y la investigación, y el buen gobierno y la rendición de cuentas en las universidades (Cátedra UNESCO, 1998).

Las actividades de la Cátedra son variadas, entre ellas se encuentran, los seminarios de formación directiva, el servicio de asesoramiento y consultoría, la investigación, las publicaciones y la cooperación internacional.

Otro elemento, a tener en cuenta, está relacionado con el hecho de que los mayores esfuerzos y estudios sobre el tema de la dirección escolar a nivel internacional, y por consiguiente de la formación jurídica como una de las áreas a tomar en cuenta en la formación integral del directivo educacional, se concentran en la educación o enseñanza general, en los niveles de enseñanza previos a la Educación Superior. En tal sentido, existe un predominio en la literatura sobre el tema de los términos directivo escolar y dirección escolar.

Lo antes expuesto, se puede corroborar a través de los trabajos de investigación y criterios de varios autores con relación a dicho tema. 
Uno de los autores de referencia en el tema de la dirección escolar en Iberoamérica es Joan Teixidó. Dicho investigador plantea, con relación a la formación de directivos escolares, cinco ideas previas o consideraciones generales. Dichas ideas 0 consideraciones son las siguientes:

1. La formación de directivos escolares (al igual que la de otros profesionales que asumen funciones complejas) tiene limitaciones. El ejercicio directivo es una tarea práctica, dinámica, contextualizada. Aunque la formación esté bien diseñada y ejecutada, no deja de ser algo artificial, alejado de la práctica cotidiana, con dificultades para dar respuestas concretas a las necesidades y expectativas de los destinatarios.

2. La eficacia de la formación aumenta en la medida que se aproxima (conoce, tiene en cuenta, da respuesta) a la problemática y los dilemas prácticos que viven los directivos. 3. Las diversas modalidades formativas (conferencias, seminarios, jornadas, talleres, trabajo en red, etc.) pueden resultar útiles en función de los objetivos y de las posibilidades organizativas. Parece lógico tender a la complementariedad y a la interrelación entre ellas.

4. Durante los últimos veinte años se ha producido una evolución positiva de la formación de los directivos escolares. Existe consciencia de su necesidad, se le destinan recursos, se diseñan planes y programas, se llevan a cabo acciones de formación de formadores, etc.

5. Existe notable consenso ante la conveniencia de avanzar desde una formación expositiva, centrada en la transmisión de información (a menudo, comentario de la normativa), hacia una formación práctico-reflexiva, que fomente la participación y la intervención activa de los asistentes y, por tanto, contribuya a su desarrollo profesional. Se trata de una modalidad que plantea mayores exigencias (a los organizadores, a los formadores y a los asistentes), pero proporciona mejores resultados en términos de capacitación para el paso a la acción (adquisición/desarrollo de competencias directivas) y, también, de satisfacción de los participantes (Teixidó, 2011, p.1-2).

Dichas ideas pueden ser tenidas en cuenta en la formación de directivos de la Educación Superior, sin obviar las particularidades que presenta el contexto universitario.

Otra estudiosa del tema, Ana María Gómez (2011), plantea que “la dirección escolar se presenta en la literatura como un factor determinante, en la mejora de los centros educativos y en los resultados escolares del alumnado, por lo que la formación para las 
tareas directivas es fundamental, para adquirir mayor competencia en el ejercicio del cargo" (Gómez, 2011, p.1).

Por su parte, Luis Villa (1998) al referirse a los objetivos de la formación de directivos escolares expresa que, tanto en este caso, como los referidos a la formación de profesores en general, nos obligan a la reflexión, acerca de los procesos de aprendizaje, y a no perder de vista el desarrollo profesional al que se aspira, a través de las actividades programadas para ello, entendiendo por desarrollo profesional, ese proceso de formación que mejora y cambia la forma de pensar y actuar; es decir, se trata de vincular las necesidades individuales a las institucionales(Villa, 1998, pp. 65-108).

Y es que, a criterio Fernández (2002, p.9), lo que debe buscarse con la formación es capacitar a los directivos escolares para las competencias que se le pidan en cada momento, de forma que comprenda cada problema que se le presente, lo valore con actitud reflexiva y crítica, y sea capaz de generar respuestas adecuadas a las necesidades de la institución.

Otro autor de referencia es Guy Pelletier, quien ha escrito numerosos artículos relacionados con la dirección escolar y la formación de los dirigentes de la educación. Una de sus obras fue publicada en 1999 y traducida posteriormente en 2003 al español, la cual se titula Formar los dirigentes de la educación. Aprendizaje en la acción. En dicha obra, ofrece una clasificación de los programas de formación atendiendo a tres categorías, asociadas a una lógica o paradigma. El primero de ellos se clasifica atendiendo a una lógica cultural, el segundo a una lógica funcional y el tercero a una lógica profesional. Este último resulta de gran interés para la formación de directivos. Siguiendo este paradigma, la formación debe ser adaptada a las exigencias de una actividad socio profesional dada. Por tanto, es importante identificar los actos profesionales, sus fundamentos, sus aspectos técnicos, éticos y estéticos, y prever un conjunto de actividades de formación, tanto teóricas como prácticas, permitiendo a los futuros dirigentes dominar la base de conocimientos y habilidades que estructuran el acto profesional, así como reconocer y compartir los valores de la profesión( Pelletier, 2003).

Además de estas investigaciones, se destacan las realizadas por la Red de Apoyo a la Gestión Educativa (RED AGE), con sede en España. Dicha organización fue creada con el propósito fundamental de fomentar el intercambio de experiencias, la profesionalización de los directivos y la promoción del conocimiento sobre administración y gestión educativa. Y su finalidad última, es la de mejorar el funcionamiento de las instituciones formativas y a través de ellas, de los sistemas formativos, procurando sean de calidad y 
un instrumento para el cambio profesional y social. Como frutos de este trabajo, fueron publicadas en 2011 dos obras de carácter colectivo, en las que un numeroso grupo de especialistas, de reconocido prestigio en lberoamérica, algunos de ellos cubanos, realizaron importantes contribuciones en el área de la dirección escolar. La primera publicación, titulada, La dirección de centros educativos en Iberoamérica. Reflexiones y experiencias, bajo la coordinación de Joaquín Gairín Sallán, plantea en la introducción a la obra, un conjunto de principios de actuación que han de orientar la concreción de actuaciones y desarrollos, sirviendo a su vez como referentes para la mejora de la dirección escolar. Dichos principios, por la importancia que revisten y la posibilidad de ser asumidos en la formación de los directivos de la Educación Superior se citan a continuación:

1. La formación se entiende, como parte del desarrollo profesional de los directivos, que debe acompañarse de otras acciones estructurales y personales en el marco de las organizaciones donde desarrollan su actividad.

2. La orientación de la formación busca preparar a los directivos como agentes de cambio en sus organizaciones. Debe así focalizarse, en el centro de formación, como espacio de innovación y cambio, teniendo en cuenta los procesos de enseñanza-aprendizaje que se producen en las aulas y los retos que el sistema educativo o el sistema formativo correspondiente plantea.

3. Defiende, asimismo, un modelo de formación participativo, crítico y colaborativo, centrado en los problemas de la práctica institucional y dirigido a la mejora organizativa. El modelo a potenciar debe superar el marco estricto de actuación y ayudar al directivo a entender el porqué y el para qué de los procesos.

4. La formación debe dar respuesta a las inquietudes institucionales y personales, combinando adecuadamente las estrategias para el cambio personal y organizacional.

5. La formación debe poner énfasis en la vinculación del trabajo directivo con el trabajo de otros profesionales que intervienen en las organizaciones, así como con la realidad contextual externa.

6. La formación persigue la mejora continua, aspirando a los mayores niveles de calidad posibles.

7. La formación se plantea como una actividad necesaria y continua para adaptarse a la cambiante realidad.(Gairín, 2011, pp. 8-9) 
La segunda publicación, coordinada por Joaquín Gairín y Diego Castro, centra su atención en las competencias de los directivos escolares, y se titula, Competencias para el ejercicio de la dirección de instituciones educativas. Reflexiones y experiencias en Iberoamérica. En el texto se hace una revisión de la función directiva, el perfil, la selección, la formación, la evaluación y el desarrollo profesional en relación a las competencias. Se reconoce como bondad del texto, presentar y analizar el grado de desarrollo que las competencias directivas tienen; también, plantear retos y algunas alternativas a la situación presentada.

No obstante, la primacía que tienen las investigaciones y la abundante literatura en el tema de la formación del directivo escolar, existen algunos trabajos relacionados con la formación de directivos universitarios, uno de ellos fue publicado en 2013 en la Revista Estrategia y Gestión Universitaria, titulado "Caso práctico de la formación de directivos en contextos universitarios".

En el citado artículo, dos investigadoras españolas, de la Universidad de Barcelona, Trinidad Mentado, y de la Universidad de Las Palmas de Gran Canaria, Josefa Rodríguez, presentan una experiencia llevada a cabo con directivos de departamentos y centros universitarios, cuyo objetivo se dirige a conocer las necesidades reales en torno a la formación para el desarrollo de la acción directiva y delimitar las posibles acciones formativas. Dicha experiencia fue desarrollada con 37 directores de departamento de la Universidad de Las Palmas de Gran Canaria.

La investigación, antes mencionada, arrojó como conclusiones, que la formación orientada a las necesidades, prioridades, y estrategias de la organización debe constituir uno de los pilares a tener en cuenta en la formación de los directivos universitarios, la cual debe acompañarse de la incorporación continua de conocimientos técnicos, de capacidades y habilidades relacionadas con el ejercicio de la función directiva, el marco normativo, y las innovaciones tecnológicas.

Se precisó además que, para poder plantear una formación de directivos, es necesario tener en cuenta que la formación debe considerar la necesidad de una preparación específica y diferenciada de la del docente; debe estar centrada en aquellos roles considerados como más difíciles: líder, técnico, etc.; tiene que permitir desarrollar estrategias para llevar a cabo el trabajo directivo con éxito; y, por último, debe considerar la existencia de un modelo de dirección participativo propio, de los departamentos universitarios. 
Y, con relación al ámbito legislativo, los encuestados consideraron a este, "como una de las grandes lagunas, sobre todo, cuando han de enfrentarse a conflictos legales con otros estamentos universitarios y reconocieron la necesidad de formarse en este aspecto." (Mentado y Rodríguez, 2013, pp.14-15).

\section{El proceso de formación jurídica de los directivos en las IES, en Cuba.}

La Educación Superior cubana tuvo sus orígenes, en la fundación de la Real y Pontificia Universidad de San Gerónimo de La Habana el 5 de enero de 1728, por la Orden de los Padres Dominicos.

Desde sus inicios y hasta finales del siglo XIX, en que se mantuvo la dominación española, todas las decisiones fundamentales en la Universidad, incluyendo las reglamentaciones académicas y curriculares, eran tomadas por el gobierno de la Isla, siguiendo los designios de la metrópoli, por lo que el papel de los directivos en este sentido era reducido, cumpliendo solo funciones representativas, evidenciando una situación bastante similar a la existente hasta ese momento a nivel internacional, en cuanto a la falta de visión sobre la necesidad de brindar una formación a las autoridades universitarias, para su desempeño exitoso al frente de las instituciones universitarias.

Con el advenimiento de la República neocolonial, tampoco se produjo ningún cambio relevante en cuanto a la necesidad de formar los directivos de las universidades. A pesar de que los mismos eran reconocidos por su prestigio profesional, no se consideraba la preparación integral necesaria, acorde con las funciones directivas que ejercían.

Con el triunfo de la Revolución, en enero de 1959, se crean las condiciones ideales, para dar un salto cualitativo y cuantitativo, en pos de transformar radicalmente la Educación Superior en el país.

Un elemento de trascendental importancia, en esta etapa, lo constituyó la Reforma Universitaria del año 1962, la cual tuvo dentro de sus planteamientos de carácter estratégico, la preocupación y ocupación por la formación y superación de los profesores dentro del modelo revolucionario que se estaba construyendo, y sentó las bases, para que los directivos universitarios pudieran formarse y desarrollarse.

Otro aspecto relevante fue la creación en 1976 del Ministerio de Educación Superior, lo cual fue un paso más de avance en la consolidación de la Educación Superior. Se produce un incremento del número de instituciones adscritas al MES y comienza a gestarse una sólida red nacional de universidades. 
La Educación Superior cubana se va a desenvolver durante la década del 80 en un entorno relativamente estable, que le permite una consolidación y mejor organización de la docencia de pregrado y postgrado, la investigación científica y el rediseño del funcionamiento de las estructuras de las IES (CEPES, 2003).

Para hacer frente a estas funciones, que no solo enriquecían, sino que hacían cada vez más compleja la gestión universitaria, se trabajó en el fortalecimiento de la preparación de profesores y directivos, sustentado en primer orden, en la Política de Cuadros, en aras de garantizar la formación del personal docente y directivo idóneo (Alpízar, 2003).

A pesar de la importancia brindada a la formación de directivos universitarios durante este período, esta no poseía un carácter sistémico y contextualizado, pues se realizaba sobre la base de programas muy generales, orientados al cumplimiento de objetivos centralizados.

A inicios de la década del 90, se le confiere al Ministerio de Educación Superior el encargo estatal de la preparación y superación de los cuadros y reservas; además, se aprueba la Estrategia Nacional de Preparación y Superación de los Cuadros y Reservas, y se elaboran las primeras estrategias ramales y territoriales de preparación y superación de cuadros y reservas en los Organismos de la Administración Central del Estado (OACE). Estas acciones constituyeron el punto de partida para la estructuración de un sistema nacional de preparación y superación de los cuadros, y un paso de avance en pos de la contextualización del proceso de formación de dichos sujetos.

En el periodo que va del año 1996 al año 2000, se indica por el Comité Ejecutivo del Consejo de Ministros (CECM) la elaboración de objetivos anuales en todos los OACE y se realiza un programa de preparación de todos los cuadros en esta temática; se aprueba y pone en vigor el Código de Ética de los Cuadros del Estado, así como los Decretos Leyes 196 y 197 sobre el trabajo con los cuadros y los funcionarios. Se generaliza la Dirección por Objetivos (DPO) e introduce la planeación estratégica, la cual devino en instrumento de gran utilidad para hacer frente a los retos cada vez mayores de la Educación Superior, en la gestión de las IES.

Comienza a producirse un cambio significativo en la conciencia de todos los cuadros acerca de la importancia y la necesidad de la preparación y la superación. Esta deja de ser una tarea que hay que cumplir, para convertirse poco a poco en una necesidad. Se abre paso la concepción de la capacitación de los cuadros no como un gasto, sino como una inversión para el desarrollo (Columbié, 2003, p.5) 
Para garantizar el éxito en la práctica de dicha planeación o proyección estratégica, resultaba necesario su complementación con la Estrategia para la Preparación y Superación de los Cuadros y Reservas. Dicha Estrategia es definida por la Comisión Central de Cuadros para ser implementada en los distintos OACE, incluido el Ministerio de Educación Superior. En dicha Estrategia se prevén, organizan y articulan los diferentes aspectos y acciones esenciales que sirven de base para lograr potenciar en términos de eficacia, eficiencia y liderazgo, la gestión de todos aquellos que en las diferentes organizaciones ejercen funciones directivas.

La Estrategia antes mencionada establece dentro de sus contenidos generales, en primer lugar, la Preparación Político-Ideológica, este es su contenido fundamental; en segundo lugar, la Preparación en Defensa Nacional y Territorial y en Defensa Civil; en tercer lugar, la Preparación en Administración(esta abarca el conjunto de temáticas principales dirigidas a preparar a los cuadros y sus reservas, acorde a las funciones de los cargos para coadyuvar a elevar la eficiencia en su gestión e incluye, integradas, la Preparación en Dirección, Económica, Jurídica, así como en el uso de la Información, y otras que se determinen, a partir de su función administrativa, ejecutiva o empresarial; y en cuarto lugar la Preparación Técnico Profesional.

Como se puede apreciar, la preparación y superación jurídica constituye una de las áreas tenidas en cuenta, en la formación integral del cuadro o directivo.

Como expresara Sierra (2008, p.2), "se hace necesario, incorporar el Derecho al actuar diario de todos los que desde una posición de dirección, están obligados a tomar decisiones, las cuales deben basarse en las normas, principios y valores que informan el ordenamiento jurídico cubano."

Si bien es cierto, que la formación jurídica es parte integrante de la Preparación en Administración, junto a otras temáticas de manera integrada, una revisión al contenido específico del resto de los componentes permiten identificar aspectos o contenidos jurídicos, pudiendo afirmarse que se trata no solo de un área de formación o subcomponente dentro de otro componente formativo, sino de un componente sui generis, caracterizado por su carácter transversal, con relación al resto de los componentes de la citada estrategia.

Dicha preparación jurídica tiene como objetivo dotar a los cuadros de conocimientos y de una cultura de respeto a la Constitución, las leyes y otras normas legales que contribuyan a elevar la disciplina laboral, social y el cumplimiento de los principios éticos. Al analizar 
este objetivo se percibe, que el mismo carece de uno de los subcomponentes inherentes a todo proceso formativo, la formación de habilidades, como parte del contenido.

Un aspecto que merece ser destacado es el hecho de que en 2010, se aprobó la cuarta versión de la Estrategia Nacional de Preparación y Superación de los Cuadros y Reservas, como muestra del continuo y permanente perfeccionamiento que se realiza en aras de lograr el impacto deseado en este proceso de formación.

También resulta loable el empeño del gobierno, de preparar y superar a todos los cuadros y reservas, en diferentes temáticas (económica, jurídica, técnicas de dirección, etc.), con el objetivo de que puedan afrontar el proceso de actualización económica que vive el país, con la aprobación de los Lineamientos de la Política Económica y Social, del Partido y la Revolución en el sexto congreso del Partido Comunista de Cuba (PCC), en abril de 2011. En este sentido, fue creada la Escuela Nacional de Cuadros, y comenzaron a desarrollarse en ella, y en las escuelas provinciales del PCC, los Diplomados en Gestión Empresarial y en Administración Pública. En dichos diplomados se dedica un módulo para la formación jurídica, a partir de reconocer la necesidad que tienen los cuadros de contar con una sólida formación en esta área para el desempeño de las funciones, que le son inherentes al cargo, las que estarán revestidas de un actuar ético, y de una cultura de respeto a la ley y de preservación de la institucionalidad durante el proceso de toma de decisiones.

Si bien, la actual investigación acerca de la formación jurídica de los directivos universitarios, es pionera en Cuba, y probablemente a nivel internacional, si existen estudios cubanos acerca de la formación de directivos universitarios. En tal sentido, se destaca la investigación doctoral realizada por Raúl Alpízar, en 2003, en la cual propone un modelo de gestión para la formación y desarrollo de los directivos académicos en la Universidad de Cienfuegos.

\section{El proceso de formación jurídica de los directivos en la Universidad de Pinar del} Río. Una aproximación diagnóstica.

La Universidad de Pinar del Río, es creada en 1972, como Filial Universitaria, luego se convierte en Centro Universitario, hasta alcanzar la condición de Universidad en 1994, y a partir de septiembre de 2015, a través de un proceso de integración de varias universidades de la provincia, incrementó el número de carreras, directivos, estudiantes, profesores y personal administrativo, al asumir en su nueva estructura, la extinta 
Universidad de Ciencias Pedagógicas y la Facultad de Cultura Física. Posee una plantilla de 153 cuadros, de ella solo está cubierta la cifra de 150, distribuida de la siguiente forma, 1 cuadro directivo (correspondiente al Rector), y 149 cuadros ejecutivos, distribuidos en las Áreas centrales, Facultades, Departamentos y Centros Universitarios Municipales.

Dicha institución, en pos de alcanzar niveles de desarrollo superiores en la gestión de todos los procesos sustantivos demanda que sus cuadros y reservas se preparen y superen sistemáticamente en los diferentes contenidos que han sido establecidos por la Comisión Central de Cuadros, con la aspiración de que logren dominar cada vez más las novedosas técnicas de dirección y apliquen de manera eficiente y creadora las tecnologías que se requieran para llevar adelante los procesos que dirigen.

En tal sentido, la Universidad de Pinar del Río cuenta con una Estrategia de Preparación y Superación de los Cuadros y Reservas, la que se actualiza cada 4 años, estando vigente, la comprendida en el período 2016-2020.

El objetivo esencial de esta Estrategia es lograr promover, canalizar y potenciar con calidad la sistemática y oportuna preparación político-ideológica de los cuadros y reservas de la Universidad, y con ello garantizar el desempeño apropiado en las funciones que les competen en el quehacer diario al frente de sus colectivos. El principal propósito es que el ejercicio de la influencia consciente sobre los subordinados, rija la consolidación de los valores compartidos definidos en la proyección estratégica, y constituyan el soporte de la batalla permanente contra la corrupción, el delito, las ilegalidades y las indisciplinas sociales en aras de la defensa de la Revolución y la concreción de los resultados económicos, políticos y sociales previstos.(Estrategia de Preparación y Superación de los Cuadros y Reservas de la Universidad para el periodo 2016-2020, 2016, p.1)

Dicha Estrategia toma como punto de partida la Estrategia Nacional, al asumir su objetivo general, principios y exigencias, contenido, formas y modalidades en que se sustenta la actividad de Preparación y Superación de los Cuadros y Reservas, aunque en cierta medida, adecuados o contextualizados al entorno de la UPR.

En relación con el contenido se establecen diferentes acciones dirigidas a garantizar la formación en dichos componentes de los Cuadros y Reservas.

De manera particular, con relación a la formación jurídica, aspecto que resulta objeto de análisis, en la presente investigación, se establecen como acciones las siguientes:

- la auto preparación dirigida y controlada por el jefe, relacionado con reglamentos y normas para el trabajo, así como la legislación vigente vinculada al cargo.

- el estudio y debate de los Decretos Leyes 196 y 197 
- el estudio y debate sobre la Constitución de la República de Cuba.

Dichas acciones tienen un carácter limitado, pues su contenido reduce las materias o temáticas jurídicas que pudieran estar vinculadas con la actividad de dirección educacional. Por otra parte, la manera de concretarlas, a través de la auto preparación, si bien están a tono con los principios y formas de la Educación Avanzada, no toma en cuenta otras formas o modalidades de carácter presencial que resultan necesarias, como cursos y entrenamientos, que sí son desarrolladas en otras áreas formativas.

A partir del año 2012, comenzaron a desarrollarse en la Escuela Provincial del PCC "Abel Santamaría Cuadrado", los Diplomados en Administración Pública, y en Gestión Empresarial, coincidiendo con el inicio de la segunda edición en la Escuela Nacional de Cuadros. En la impartición de dichos diplomados en la provincia, ha desempeñado un papel fundamental el Centro de Estudios de Gerencia de la UPR como ente coordinador. Y le ha correspondido, al claustro de profesores del Departamento de Derecho, desarrollar en ambos diplomados la formación jurídica de los cuadros matriculados en cada uno de ellos.

Desde su inicio en 2012 y hasta la fecha, con la XII Edición, han egresado alrededor de 700 cuadros, entre ellos el Rector, varios vicerrectores y decanos de la UPR.

A pesar de este empeño, aún es insuficiente el número de cuadros en la UPR que reciben formación jurídica presencial. Tampoco la formación que se imparte es contextualizada al entorno de la dirección universitaria pues la misma se dirige a directivos de varios sectores de la sociedad, siendo su contenido de carácter general. No obstante, la posible solución de un problema jurídico, presente en el contexto de su organización, es orientado como evaluación final del módulo de bases jurídicas.

Los instrumentos utilizados para obtener la mayor cantidad de información sobre el objeto de investigación y constatar el problema investigado fueron:

- análisis documental (Estrategia Nacional de Preparación y Superación de los Cuadros del Estado, del Gobierno y sus Reservas aprobada en el año 2010, el Plan de Preparación y Superación de los Cuadros y Reservas de la UPR para el cuatrienio 2012-2015, así como el correspondiente al cuatrienio 2016-2020 y el Programa Anual de Preparación y Superación de los Cuadros y sus Reservas de la UPR de los años 2012, 2013, 2014, 2015 y 2016).

- encuesta a cuadros en la UPR. 
- entrevista grupal a profesores del Departamento de Derecho a tiempo completo y parcial, que participan en la capacitación jurídica de los cuadros en la provincia, incluidos los de la Educación Superior.

$\checkmark$ Análisis de documentos.

A partir de las fuentes documentales consultadas, y atendiendo a la guía elaborada, se puede precisar que en el proceso de formación jurídica de los cuadros en la UPR se evidencian problemas que se concretan en:

- el insuficiente tratamiento de la temática jurídica en la Estrategia Nacional de Preparación y Superación de los Cuadros del Estado, del Gobierno y sus Reservas, lo cual se mantiene, de manera idéntica, en la Estrategia de Preparación y Superación de Cuadros y Reservas de la UPR, demostrando falta de pertinencia y descontextualización, a la hora de concebir dicha formación.

- la limitada fundamentación pedagógica y didáctica de la Estrategia, plan y programa respectivamente, pues no se desarrollan adecuadamente en cada una de las áreas de preparación y superación, los aspectos que deben estar presentes en el contenido, como uno de los componentes didácticos de todo proceso formativo, integrado por conocimientos, habilidades, actitudes y valores. La formación jurídica identificada como preparación específica dentro del área integradora Dirección-Administración solo contempla la mera transmisión o asimilación de conocimientos, y la promoción de determinados valores, sin que se contemple la necesaria formación de habilidades.

- la insuficiente organización didáctica siguiendo la lógica de la ciencia, a la hora de concebir los contenidos del área de preparación jurídica en relación con el resto de las áreas formativas, los que se limitan exclusivamente al estudio y debate en torno a las leyes y reglamentos propios de la actividad y del cargo, incluida la Constitución de la República, con un visión reducida de los campos o áreas del Derecho que pueden tributar al desempeño eficiente del cuadro en la solución de problemas con trascendencia jurídica, que se le presentan en el contexto universitario. Tampoco se planifica ninguna acción de superación, tales como cursos, talleres, diplomados, utilizando solo la auto preparación dirigida y controlada por el jefe, lo cual difiere del resto de las áreas de formación, que si resultan privilegiadas al concebir o planificar varias formas organizativas para la preparación y superación de dichos sujetos.

Con el fin de corroborar estos problemas se procedió a la aplicación de otros instrumentos de diagnóstico, precisados anteriormente en este epígrafe. 
$\checkmark$ Análisis de las encuestas realizadas a los cuadros en la UPR.

Después de aplicada la encuesta a una muestra de 107 cuadros, se pudo constatar que:

- El $40 \%$ de los encuestados desconocen la Estrategia de la Universidad para la preparación y superación de los cuadros y reservas.

- El $53 \%$ de los encuestados consideran que su nivel de formación jurídica es bajo. Se alega, como causa general, una insuficiente atención de los temas jurídicos en las capacitaciones o cursos que se brindan a los cuadros.

- El $70 \%$ de los encuestados tienen una visión limitada con relación al contenido de la formación jurídica, pues solo la asocian al estudio y conocimiento de las leyes.

- Solo el $28 \%$ de los encuestados declara haber recibido alguna formación jurídica, la cual ha sido desarrollada en alguna medida, en los espacios generales de debates y reflexiones, las conferencias impartidas a los cuadros y profesores, los Consejos de Dirección y los Colectivos de Dirección Docente.

- El $73 \%$ de los que declaran haber recibido formación jurídica, expresan la falta de sistematicidad, pues la frecuencia aproximada con que reciben este tipo de formación es baja (una vez en cada curso académico).

- El $84 \%$ de los que declaran haber recibido formación jurídica consideran que no se contextualiza al perfil del desempeño de cada cuadro.

- El 97 \% reconoce la importancia de la formación jurídica como componente de la Estrategia para su preparación integral y desempeño profesional en el cargo. En tal sentido destacan que:

-da mayor certeza a la hora de evaluar las decisiones que se adoptan, además de que estarán impregnadas de una mayor justicia.

-apoya la docencia y el Trabajo Político Ideológico.

-enseña no solo el contenido de una norma sino que permite su correcta interpretación y/o aplicación.

Se demanda además por los encuestados, la necesidad de disponer de una oferta de cursos de superación en materia jurídica, que les brinde una formación integral, así como contar con espacios de consulta y de asesoría jurídica.

- Los encuestados manifiestan el interés de que se incluya en un Programa de Formación Jurídica dirigido a su formación integral, aspectos de carácter general, que deben ser tenidos en cuenta por todos los cuadros de la Educación Superior y 
otros de carácter específico, acorde al perfil del desempeño de cada cuadro. En tal sentido proponen las siguientes temáticas:

$\checkmark$ Análisis del Código de Ética de los Cuadros del Estado.

$\checkmark$ Nociones básicas sobre Derecho Constitucional (Sistema Político Cubano, Sistema Electoral, derechos y deberes de los ciudadanos).

$\checkmark$ Reglamentos de la Educación Superior (Disciplinario, Trabajo Docente Metodológico, Organización Docente, etc.), y demás disposiciones internas del MES.

$\checkmark$ Redacción de instrumentos jurídicos (Reglamentos, Resoluciones, Contratos, Convenios).

$\checkmark$ Algunas cuestiones relacionadas con el Derecho Laboral.

$\checkmark$ Nociones sobre la Propiedad Intelectual.

- El $90 \%$ de los encuestados ubicó la formación jurídica como área prioritaria en su formación como cuadro.

$\checkmark$ Las vías que consideran más útiles para su formación, son la formación en el puesto de trabajo, la auto superación y el adiestramiento.

$\checkmark$ Los encuestados consideran que los aspectos que deben ser tenidos en cuenta para elevar su formación jurídica y perfeccionar su trabajo son: la capacitación jurídica y la asesoría jurídica de expertos.

Los resultados antes expuestos permiten constatar la insuficiente formación jurídica de los cuadros en la Universidad de Pinar del Río, además de su carácter asistemático, dada las esporádicas acciones de formación que se llevan a cabo en este sentido. Se evidencia además una desarticulación y una descontextualización, así como una limitada fundamentación pedagógica y didáctica.

$\checkmark$ Entrevista grupal a 8 profesores del Departamento de Derecho.

La entrevista grupal arrojó el reconocimiento de los profesores, de la necesidad de una capacitación específica para enfrentar la formación de los cuadros, por sus características propias como sujetos adultos, y porque implica, según el perfil de su desempeño y el contexto en el que se desenvuelve, el dominio de un gran número de contenidos, presentes en la mayoría de las ramas del Derecho. Se pudo constatar, además, que los profesores que participan en la formación de los cuadros poseen cierta experiencia 
pedagógica, avalada por más de 8 años en el ejercicio de la docencia, varios de ellos con estudios de postgrado en ciencias de la educación.

Los entrevistados coinciden en que las insuficiencias, que presenta actualmente el proceso de formación jurídica de los cuadros, radican en su descontextualización, alejado de las necesidades específicas en la UPR, y en cuanto a su concepción pedagógica y didáctica con relación al contenido, al no estar definidas las habilidades a formar, y por consiguiente no darse la integración entre los tres componentes (conocimientos, habilidades y valores), por lo que carece de un enfoque sistémico y coherente.

Sugieren para cambiar la situación existente, fundamentar desde las Ciencias de la Educación una propuesta, que permita concebir ese proceso, de forma sistémica y contextualizada, además de recibir ellos una capacitación para la formación de los cuadros, dadas sus particularidades, que les permita enfrentar el proceso de formación jurídica de dichos sujetos en la UPR.

El análisis, interpretación e integración de estos resultados, permiten determinar las siguientes regularidades en el proceso de formación jurídica de los cuadros en la UPR.

1. El proceso de formación jurídica de los cuadros en la UPR, no está dirigido a la formación y desarrollo de habilidades, para la solución de problemas, con trascendencia jurídica, que se presentan en el contexto universitario, durante su desempeño profesional, acorde al perfil de su cargo, lo que se demuestra en las siguientes dificultades:

-No se identifican las habilidades a formar en los cuadros, para contribuir a su desempeño profesional en el cargo y a la solución de problemas con trascendencia jurídica presentes en el contexto universitario.

-Los cuadros tienen una visión limitada acerca del Contenido de la formación jurídica.

2. El contenido del proceso de formación jurídica de los cuadros en la UPR, no garantiza su carácter sistémico y contextualizado.

3. Existen insuficiencias en la preparación teórico- metodológica de los profesores del Departamento de Derecho para asumir el proceso de formación jurídica de los cuadros, con un carácter sistémico y contextualizado, dada las características propias de esta formación. 


\section{CONCLUSIONES}

La sistematización de las principales ideas abordadas en este trabajo, converge en la necesidad de desarrollar un proceso de formación jurídica de los directivos, en las IES, sistémico y contextualizado, de manera que garantice una mayor relación de su preparación profesional con el impacto de su desempeño profesional en el cargo. Estas ideas se resumen en que:

- la formación jurídica de los directivos en las IES transitó por un largo período, en que no era considerada ni atendida como se requería la preparación de los directivos de las instituciones universitarias, hasta el reconocimiento en los últimos años de la necesidad de formar a estos sujetos en pos de potenciar su preparación integral para su desempeño profesional en el cargo.

- el escaso número de trabajos dirigidos a analizar el papel de la formación de los directivos en las IES, y por consiguiente de la formación jurídica, en comparación con el notable aumento de los estudios que sobre la temática de la formación de directivos escolares se llevan a cabo, denota la necesidad de brindar una mayor atención y prioridad al análisis y desarrollo de la formación de los directivos en las IES.

- la aplicación de métodos empíricos revela, que el proceso de formación jurídica de los cuadros en la UPR no favorece la formación de habilidades y por tanto, no se concibe como proceso que debe contribuir a la solución de problemas con trascendencia jurídica durante su desempeño profesional, acorde al perfil de su cargo.

Revela, además, insuficiencias en la preparación teórico-metodológica de los profesores de la carrera para asumir el proceso de formación jurídica, de manera sistémica y contextualizada, dadas las características propias de esta formación.

\section{REFERENCIAS BIBLIOGRÁFICAS}

Alpízar, R. (2003). Modelo de gestión para la formación y desarrollo de los directivos académicos en la Universidad de Cienfuegos. Tesis doctoral. La Habana: CEPES.

Álvarez, C. M. (1999). Didáctica. La escuela en la vida. Tercera edición. La Habana: Editorial Pueblo y Educación.

Cánova, A., Cruz, L. \& Vecino, U. (2016). Apuntes sobre la superación profesional en instituciones de la Educación Superior. Revista Educación y Sociedad, 14(3), pp.3850 . 
Colectivo de Autores. (1984). Pedagogía. La Habana: Editorial Pueblo y Educación.

Columbié, M (2003). Momentos significativos de la preparación y superación de los cuadros en Cuba a partir del triunfo de la Revolución. Recuperado el 7 de mayo de 2015.http://folletosg.gob.edu.cu

Fernández, M.L. (2002). Formar para la dirección escolar: Por qué, cuándo, cómo...Profesorado, revista de curriculum y formación del profesorado, 6 (1-2).p.9.

Figueroa, V., Pliscoff, C. \& Araya, JP. (2014). Desafíos a la formación de los futuros directivos públicos del siglo XXI. Convergencia Revista de Ciencias Sociales, núm.64. pp. 207234. Toluca, México: Universidad Autónoma del Estado de México.

Gairín, J. (2011). La Dirección de Centros Educativos en Iberoamérica. Reflexiones y experiencias. Serie Informes 2. Red de Apoyo a la Gestión Educativa. Recuperado el 2 de diciembre de 2013.

http://www.redage.org/files/adjuntos/Libro\%20Red_AGE\%20vd.pdf

Gairín, J y Castro, D. (2011). Competencias para el Ejercicio de la Dirección de Instituciones Educativas. Reflexiones y experiencias. Serie Informes 2. Red de Apoyo a la Gestión Educativa. Recuperado el 3 de diciembre de 2013. http://accelera.uab.cat/documents_edo/biblio/Redage2012.pdf.

Gómez, A.M. (2010). La formación inicial para la dirección escolar como impulso para conseguir una dirección competente en Andalucía. Memoria para optar al grado de doctora. Huelva, España.

Horruitiner, P. (2007). La Universidad cubana: el modelo de formación. Editorial Félix Varela, La Habana.

Koontz \& O'Donell. (1961). Principios de dirección de empresas. Madrid: McGrawHill/Ediciones del Castillo. pp. 375-379. 
López, J.C \& Pérez, I. (2015). Gestión de la formación de directivos y su vínculo con la práctica organizacional. Propuesta de modelo contextual. Revista Ecociencia, 2(3). pp. 1-21. Ecuador.

Mentado, T. \& Rodríguez, J. (2013). Caso práctico de la formación de directivos en contextos universitarios. Revista Estrategia y Gestión Universitaria. España.pp.14-15.

Rodríguez, J., Artiles, J., Aguiar, M.V. (2015). La gestión universitaria: dificultades en el desempeño del cargo y necesidades de formación. Revista de Docencia Universitaria, 13(3), pp. 213-231.

Rodríguez, J., Artiles, J. (2017). Aprendizajes y Buenas Prácticas para la Gestión de la Institución Superior. Revista Iberoamericana sobre Calidad, Eficacia y Cambio en Educación, 15(1), pp.129-141.

Rosental, M. \& ludin, P. (1981). Diccionario Filosófico. Editora Política, La Habana, Cuba.

Sierra, J. (2004). La Educación Jurídica. Propuesta de un sistema de trabajo teórico y metodológico para la formación inicial y permanente de maestros primarios. Tesis doctoral. Pinar del Río, Cuba. p.48.

- (2008). El componente jurídico del sistema normativo del Ministerio de Educación. Pinar del Río, Cuba. p.2.

Siliceo, A. (1981). Capacitación y Desarrollo de Personal. México: Limusa.p.47.

Teixidó, J (2012). La formatió de directius escolars. Fundació Universitat de Girona: Innovació y Formatió. España .Recuperado por correo electrónico.pp.1-2.

Tunnermann, C. (1996). La Educación Superior en el umbral del Siglo XXI. Caracas. Ediciones CRESAL/UNESCO.

UPR. (2016). Estrategia de Preparación y Superación de los Cuadros y Reservas de la Universidad para el periodo 2016-2020.p.1. 
Villa, A. (1998). El rol del liderazgo en la dirección actual. Principales dificultades de la dirección de centros educativos en los primeros años de ejercicio. Bilbao. ICE. Universidad de Deusto.pp.65-108.

Licencia Creative Commons

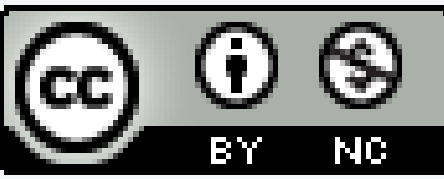

Revista Científica ECOCIENCIA está bajo una Licencia Creative Commons Atribución-NoComercial 4.0 Internacional. 